\title{
A study on the method of short-time approximation - Criteria for applicability
}

\author{
Alvin Casandra ${ }^{a}$, Suryadi Ismadji ${ }^{b}$, Boris A. Noskov ${ }^{c}$, Libero Liggieri ${ }^{d}$, Shi-Yow Lin $^{\mathrm{a}, *}$ \\ a Department of Chemical Engineering, National Taiwan University of Science and Technology, 43, Keelung Road, Sec. 4, Taipei 106, Taiwan \\ ${ }^{\mathrm{b}}$ Department of Chemical Engineering, Widya Mandala Catholic University, 37, Kalijudan, Surabaya, Indonesia \\ ${ }^{\mathrm{c}}$ Department of Colloid Chemistry, St. Petersburg State University, Universitetsky pr. 26, 198504 St. Petersburg, Russia \\ ${ }^{\mathrm{d}}$ CNR - Istituto per l'Energetica e le Interfasi IENI - UOS Genova, via De Marini, 6 Genova, Italy
}

\section{A R T I C L E I N F O}

\section{Article history:}

Received 14 November 2014

Received in revised form 25 June 2015

Accepted 6 July 2015

\section{Keywords:}

Surfactant

Short-time approximation

Diffusivity

Dynamic surface tension

Ward-Tordai equation

Planar surface

\begin{abstract}
A B S T R A C T
Despite its widespread use in the determination of adsorption mechanisms and the estimation of surfactant diffusivity, the short-time approximation method, used for linearly fitting experimental dynamic surface tension data, should be validly applied only over a very specific range of time intervals or surface pressures. Therefore, the definition of general criteria for the applicability of this method and for error evaluation in diffusivity estimations is fundamental. In this work, a theoretical numerical simulation of the short-time approximation method was conducted, and general benchmarks for its accurate utilization were investigated. Specifically, for systems assuming planar gas-liquid surfaces, diffusion-controlled kinetics and a Langmuir adsorption isotherm, simple rules were developed in terms of limiting surface pressure $\left(\pi_{\max }\right)$ and dimensionless time $\left(t_{\max }^{*}\right)$ as a function of dimensionless surfactant concentration $\left(C_{0} / a\right)$. For values greater than the limiting (maximal) conditions, the dynamic surface tension curve deviates from the short-time approximation straight line, and thus, the corresponding linear fitting could lead to significant errors in evaluating the diffusivity. The simple criteria proposed in this study thus precisely define the range of applicability for the short-time approximation method.
\end{abstract}

(c) 2015 Elsevier Ltd. All rights reserved.

\section{Introduction}

Surfactants are essential agents in sundry practical applications and products, including detergents, inks, adhesives, pesticides, and cosmetics [1]. In recent years, the global market for surfactants has shown rapid growth, with a 3.8\% annual increase: in 2012, the market value was estimated at approximately US $\$ 26.8$ billion, and it is expected to reach US\$31.1 billion by 2016 [2]. Because surfactant optimization depends on specific knowledge of their dynamic adsorption behavior [3], it is not surprising that this field of study has gained tremendous attention over the past two decades.

In 1946, Ward and Tordai introduced a general equation for interpreting the surfactant adsorption kinetics of planar gas-liquid surfaces [4]. In their model, the authors assumed that surfactant diffusion from the bulk to the sublayer is the limiting step compared to surfactant transfer from the sublayer to the surface. This diffusion-controlled approach has been observed to be valid for

\footnotetext{
* Corresponding author. Tel.: +88622737 6648; fax: +8862 27376644

E-mail address: sylin@mail.ntust.edu.tw (S.-Y. Lin).
}

the majority of small, pure surfactants and surfactant mixture systems [5] such that the equation describing their behavior is considered a fundamental starting point in numerous models used today.

However, the Ward-Tordai equation is rather complex, and its application has been hindered for decades by its complicated numerical calculations. Therefore, in 1959, Defay and Hommelen [6] and Hansen and Wallace [7] introduced a simplified form of the equation, assuming that surfactant backward diffusion from the sublayer to the bulk solution could be omitted for initial short time intervals. In 1979, van den Bogaent and Joos [8] coupled this simplified equation with the Gibb's adsorption equation and von Szyszkowski's equation, developing an easy-to-use linear approximation for the Ward-Tordai equation.

Due to its simplicity, this short-time linear approximation equation has been widely used to date [9-41]. Specifically, many researchers $[10,14-17,19,20,22,23,25,27-32,34,35,37,38,40]$ have utilized the equation to evaluate surfactant diffusivities or determine the adsorption mechanism of various systems, linearly fitting dynamic surface tension data with the short-time linear approximation equation ( $\gamma$ vs. $t^{1 / 2}$ ) for specific ranges of "short" time intervals (generally $t^{1 / 2}=0-5 s^{1 / 2}$, but up to $t^{1 / 2}=200 s^{1 / 2}$ in [22]). A 
detailed literature review of these diffusivities, estimated by using the short-time approximation method, is reported in Table 1.

Intuitively, because dynamic surface tension data can be accurately linearly fitted only for specific initial time intervals, an appropriate choice of the time range plays a crucial role in the correct evaluation of diffusivity. In fact, it has been reported $[22,23,25,28]$ that quite diverse values of diffusivity have been observed when different time ranges were chosen. Ultimately, thus, certain general criteria for validly applying the short-time approximation method would be essential to guiding researchers in correctly estimating diffusivities.

Therefore, in this work, a theoretical numerical simulation of the short-time approximation method was conducted, and general benchmarks for its accurate utilization were investigated. All calculations were carried out for a diffusion-controlled adsorption process involving the mass transport of surfactant molecules from a uniform bulk phase to a freshly created air-water interface. The following conditions were applied: (1) a Langmuir adsorption isotherm, (2) a planar air-water interface, (3) room temperature $25^{\circ} \mathrm{C}$, and (4) a diffusivity of $D_{\text {set }}=5 \times 10^{-6} \mathrm{~cm}^{2} / \mathrm{s}$ for different dimensionless surfactant concentrations (the ratio between the bulk concentration and the surfactant activity) $C_{0} / a$. Additional simulations were conducted for different $a$ values.

\section{Theoretical framework}

\subsection{Ward-Tordai equation}

In the case of one-dimensional diffusion and adsorption onto a planar surface from a bulk phase initially containing a uniform distribution of a surface active solute, the diffusion of the surfactant in the bulk continuous phase is described by Fick's law:

$\frac{\partial C}{\partial t}=D \frac{\partial^{2} C}{\partial z^{2}}(z>0, t>0)$

where $z$ is the distance from the surface and $C(z, t)$ is the bulk concentration of surfactant molecules. The boundary and initial conditions for Eq. (1) are as follows:

$$
\begin{aligned}
& C(z, t)=C_{0}(z>0, t=0) \\
& C(z, t)=C_{0}(z \rightarrow \infty, t>0) \\
& \frac{\mathrm{d} \Gamma}{\mathrm{d} t}=D \frac{\partial C}{\partial z}(z=0, t>0) \\
& \Gamma(t)=0(t=0)
\end{aligned}
$$

\begin{tabular}{|c|c|c|c|c|c|c|c|}
\hline \multirow[t]{2}{*}{ Refs } & \multirow[t]{2}{*}{ Compound } & \multirow[t]{2}{*}{$D\left(10^{-6} \mathrm{~cm}^{2} / \mathrm{s}\right)$} & \multirow[t]{2}{*}{$C_{0}\left(\mathrm{~mol} / \mathrm{cm}^{3}\right)$} & \multirow[t]{2}{*}{$t^{1 / 2}\left(\mathrm{~s}^{1 / 2}\right)$} & \multirow[t]{2}{*}{$\pi^{\mathrm{a}}(\mathrm{mN} / \mathrm{m})$} & \multicolumn{2}{|l|}{ Model parameter $^{\mathrm{e}}$} \\
\hline & & & & & & $\overline{\Gamma \infty\left(10^{-10} \mathrm{~mol} / \mathrm{cm}^{2}\right)}$ & $a\left(10^{-10} \mathrm{~mol} / \mathrm{cm}^{3}\right)$ \\
\hline \multirow[t]{2}{*}{13} & $\mathrm{Glu}_{8}-2-\mathrm{Glu}_{8}$ & 1.5 & $3.5 \times 10^{-6}$ & $0.1-0.3$ & 6.0 & 3.99 & 4.23 \\
\hline & $\mathrm{Glu}_{12}-2-\mathrm{Glu}_{12}$ & 0.17 & & $0.1-0.2$ & 0.6 & 4.39 & 0.31 \\
\hline \multirow[t]{2}{*}{16} & FluoroCarbon $_{4}$ & 2.3 & $1 \times 10^{-8}$ & $0-14$ & 6.0 & 10.0 & 63.8 \\
\hline & & & $2 \times 10^{-8}$ & $0-14$ & 13 & & \\
\hline 17 & $\mathrm{C}_{9}-\mathrm{TGE}^{\mathrm{b}}$ & 1.1 & $2.6 \times 10^{-8}$ & $0-3$ & 3.5 & 2.46 & 8.06 \\
\hline 19 & $\mathrm{C}_{12}$-Maltose ester & 3.4 & $0.8 \times 10^{-6}$ & $0-0.3$ & 3.0 & 3.34 & 55.8 \\
\hline \multirow[t]{2}{*}{20} & ANHG550 ${ }^{\mathrm{C}}$ & 2.4 & $0.32 \times 10^{-6}$ & $0-0.65$ & 2.0 & 2.13 & 4.40 \\
\hline & & 2.6 & $1.2 \times 10^{-6}$ & $0-0.2$ & 10 & & \\
\hline \multirow[t]{2}{*}{22} & Pluronic F68 & 0.92 & $0.17 \times 10^{-11}$ & $0-200$ & 3.0 & 4.84 & 0.021 \\
\hline & & 0.92 & $0.72 \times 10^{-11}$ & $0-30$ & 4.0 & & \\
\hline 23 & $\mathrm{C}_{10} \mathrm{E}_{6}$ & 57 & $8 \times 10^{-8}$ & $0-0.4$ & 22 & 2.30 & 10.8 \\
\hline 25 & $\mathrm{di}-\mathrm{C}_{8}$ & 3.0 & $2 \times 10^{-7}$ & $0-0.55$ & 7.0 & 2.81 & 1.68 \\
\hline \multirow[t]{4}{*}{27} & $\mathrm{C}_{8} \mathrm{DMPO}^{\mathrm{d}}$ & 5.3 & $4 \times 10^{-7}$ & $0-0.09$ & 2.0 & 3.20 & $3830^{\mathrm{e}}$ \\
\hline & & & $20 \times 10^{-7}$ & $0-0.05$ & 7.5 & & \\
\hline & $\mathrm{C}_{10} \mathrm{DMPO}{ }^{\mathrm{d}}$ & 4.8 & $2 \times 10^{-7}$ & $0-0.2$ & 1.5 & 3.60 & $420^{e}$ \\
\hline & & & $20 \times 10^{-7}$ & $0-0.05$ & 17 & & \\
\hline \multirow[t]{2}{*}{28} & $\mathrm{C}_{10} \mathrm{E}_{4}$ & 4.9 & $0.05 \times 10^{-6}$ & $0-1.6$ & 2.0 & 3.18 & 25.7 \\
\hline & & & $0.6 \times 10^{-6}$ & $0-0.9$ & 17 & & \\
\hline \multirow[t]{4}{*}{29} & b-lactoglobulin & 4.9 & $0.1 \times 10^{-9}$ & $0-30$ & 0.5 & 1.88 & 2.02 \\
\hline & & & $5 \times 10^{-9}$ & $0-12$ & 17 & & \\
\hline & b-casein & 4.9 & $0.1 \times 10^{-9}$ & $0-30$ & 1.0 & 4.87 & 0.77 \\
\hline & & & $5 \times 10^{-9}$ & $0-30$ & 14 & & \\
\hline \multirow[t]{2}{*}{30} & DPPC & 12 & $2 \times 10^{-9}$ & $0-19$ & 5.8 & 2.79 & $2.71^{\mathrm{e}}$ \\
\hline & & 8 & $10 \times 10^{-9}$ & $0-17$ & 15 & & \\
\hline \multirow[t]{2}{*}{31} & Hexanol & 1.5 & $5 \times 10^{-6}$ & $0-0.07$ & 9.0 & 6.86 & 52100 \\
\hline & & 0.39 & $10 \times 10^{-6}$ & $0-0.03$ & 12 & & \\
\hline 32 & MTAB & 13.4 & $4 \times 10^{-8}$ & $0-1$ & 5.9 & 2.80 & $3600^{e}$ \\
\hline \multirow[t]{2}{*}{33} & Triton X-405 & 0.7 & $2.54 \times 10^{-8}$ & $0-0.21$ & 0.1 & 0.92 & 0.22 \\
\hline & & & $7.63 \times 10^{-8}$ & $0-0.20$ & 1.0 & & \\
\hline 34 & TDSNa & 2.0 & $2 \times 10^{-6}$ & $0-0.09$ & 14 & 9.24 & $4030^{\mathrm{e}}$ \\
\hline \multirow[t]{2}{*}{35} & SDS & 4.7 & $0.5 \times 10^{-6}$ & $0-3$ & 4.0 & 3.84 & $16000^{\mathrm{e}}$ \\
\hline & & 2.8 & $2 \times 10^{-6}$ & $0-3.1$ & 12 & & \\
\hline 37 & Na-myristate + Na-Decanoate & 1.1 & $2.25 \times 10^{-7}+1 \times 10^{-5}$ & $0-1$ & 7.3 & 3.08 & $38800^{\mathrm{e}}$ \\
\hline \multirow[t]{4}{*}{38,40} & $\mathrm{DC}_{10} \mathrm{PO}$ & 0.029 & $0.29 \times 10^{-7}$ & $0-8$ & 4.4 & 3.70 & $330^{\mathrm{e}}$ \\
\hline & & 0.011 & $1.1 \times 10^{-7}$ & $0-6$ & 11 & & \\
\hline & $\mathrm{DC}_{12} \mathrm{AO}$ & 0.4 & $4.37 \times 10^{-8}$ & $0-10$ & 7.2 & 4.20 & $280^{\mathrm{e}}$ \\
\hline & & & $17 \times 10^{-8}$ & $0-8$ & 15 & & \\
\hline
\end{tabular}

Table 1

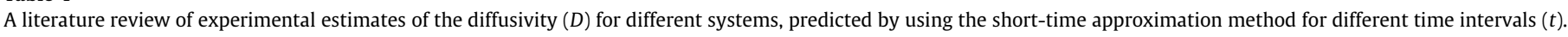

a Surface pressure, $\pi=\gamma_{0}-\gamma$.

b $\mathrm{TGE}=$ tryptophan glycerol ether surfactant.

c ANHG550 = heterogeminis surfactant of PEG with MW $=550$.

d DMPO = dimethyl phosphine oxides.

e Parameters ( $\Gamma \infty$ and $a$; maximum surface concentration and surfactant activity) obtained from previous studies. 
where $C_{0}$ is the initial bulk concentration, $\Gamma$ is the surface concentration of surfactant and zero $\Gamma$ indicates an initially clean air-water surface.

By using the Laplace transform, the adsorption of surfactant molecules as a function of time can be formulated in terms of an unknown sublayer concentration $C_{\mathrm{S}}(t)=C(z=0, t)$ :

$\Gamma(t)=2 C_{0}\left(\frac{D t}{\pi}\right)^{1 / 2}-2\left(\frac{D}{\pi}\right)^{1 / 2} \int_{0}^{\sqrt{t}} C_{S}(t-\tau) d \sqrt{\tau}$

where $C_{\mathrm{S}}$ is the sublayer concentration and $D$ is the surfactant diffusivity. This relation is the well-known Ward-Tordai equation, which is applicable for an initially clean planar gas-liquid surface and a diffusion-controlled surfactant mass transport process. This sublayer concentration can be determined numerically [42] by a modification of the technique used by Miller and Kretzschmar [43].

Specifically, the first and second terms on the right-hand side of Eq. (6), defined in this work as Term $_{F}$ and $\mathrm{Term}_{B}$, are linked to surfactant forward diffusion from the bulk phase to the sublayer and surfactant backward diffusion from the sub-layer to the bulk solution, respectively.

\subsection{Dynamic surface tension}

To obtain the theoretical dynamic surface tension curve, the Ward-Tordai equation (6) has to be combined with two additional relations: the adsorption isotherm and the equation of state.

The adsorption isotherm relates the surface concentration and subsurface concentration of surfactant molecules at constant temperature. When it is assumed that the mass transport process is diffusion-controlled and the adsorbed surfactants on the surface do not interact with each other, the Langmuir isotherm can describe the relationship between the surface concentration and the bulk surfactant concentration:

$\frac{\Gamma}{\Gamma_{\infty}}=x=\frac{C}{a+C}$

where $\Gamma_{\infty}$ and $a$ are the model parameters of the Langmuir isotherm, and $x$ is the dimensionless surface concentration. Parameter $\Gamma_{\infty}$ is the maximum surface concentration and $a$ indicates the surfactant activity. The Ward-Tordai equation (6) coupled with the Langmuir adsorption isotherm (Eq. (7)) can be solved numerically to obtain $\Gamma(t)$.

Finally, the equation of state determines the dependence of the surface tension on the surface concentration:

$\gamma(t)=\gamma_{0}+R T \Gamma_{\infty} \ln \left[\Gamma(t) / \Gamma_{\infty}\right]$

where $R$ is the universal gas constant, $T$ is temperature and $\gamma_{0}$ is the surface tension of solvent.

\subsection{Short-time linear approximation method}

At the beginning of the adsorption process $(t \sim 0)$, surfactant backward diffusion from the sublayer to the bulk phase can be assumed to be negligible, such that $\mathrm{Term}_{B}$ of the Ward-Tordai equation (6) can be omitted, and the surfactant surface concentration $\Gamma$ at a time $t$ is given by

$\Gamma(t)=2 C_{0} \sqrt{\frac{D t}{\pi}}$

The Gibbs adsorption equation relates the surface tension $(\gamma)$ to the surface concentration $(\Gamma)$ :

$d \gamma=-R T \Gamma \operatorname{dln} C$

Integrating Eq. (10) and applying Eq. (9) yields the following: $\int_{0}^{\gamma} \mathrm{d} \gamma=-\int_{0}^{C_{\mathrm{S}}} \frac{R T \Gamma}{C_{\mathrm{S}}} \mathrm{d} C_{\mathrm{S}}=-2 C_{0} R T \sqrt{\frac{D}{\pi}} \int_{0}^{C_{\mathrm{S}}} \frac{\sqrt{t}}{C_{\mathrm{S}}} \mathrm{d} C_{\mathrm{S}}$

If the term $\sqrt{t} C_{S}$ is considered to be constant during the initial stage of the adsorption process with initial condition (5) $\left[\Gamma(t=0)=0\right.$, i.e., $\left.C_{S}(t=0)=0\right]$, the following relation is obtained:

$\gamma(t)-\gamma_{0}=-2 C_{0} R T \sqrt{\frac{D}{\pi}} \frac{\sqrt{t}}{C_{\mathrm{S}}} \int_{0}^{C_{\mathrm{S}}} \mathrm{d} C_{\mathrm{S}}=-2 C_{0} R T \sqrt{\frac{D t}{\pi}}$

which leads to the so-called short-time approximation equation and a linear relationship between $\gamma(t)$ and $t^{1 / 2}$. It should be noted that Eq. (12) holds for a diffusion-controlled adsorption process.

As illustrated in the introduction, for decades, Eq. (12) has been used by many researchers $[10,14-17,19,20,22,23,25,27-32,34,35$, $37,38,40$ ] as an easy method for determining a diffusioncontrolled adsorption process and for estimating surfactant diffusivity. A linear dependence between $\gamma(t)$ and $t^{1 / 2}$ is commonly used to determine whether an adsorption process is diffusion-controlled [8], and the surfactant diffusivity is estimated by simply linearly fitting the initial slope of the $\gamma(t)$ curve in the $\gamma(t)-t^{1 / 2}$ plot by setting this slope equal to $2 R T C_{0} \sqrt{D / \pi}$.

\section{Results}

To determine the valid ranges of applicability of the short-time approximation technique for identifying adsorption mechanisms and estimating diffusivity, a theoretical numerical simulation was performed in this study. All calculations were carried out for an established diffusivity of $D_{\text {set }}=5 \times 10^{-6} \mathrm{~cm}^{2} / \mathrm{s}$ and for different dimensionless bulk concentrations $C_{0} / a$.

Initially, by using the Ward-Tordai equation and the Langmuir adsorption isotherm, with parameters $\Gamma_{\infty}=5 \times 10^{-10} \mathrm{~mol} / \mathrm{cm}^{2}$ and $a=1 \times 10^{-9} \mathrm{~mol} / \mathrm{cm}^{3}$ as delineated in Section 2, the exact relaxation curves of the dynamic surface tension, $\gamma(t)$, and the surface concentration, $\Gamma$, were generated for five different values of $C_{0} / a$, as shown in Fig. 1a. Plotting the surface tension (or surface pressure) as a function of $t^{1 / 2}$, initially linear trends (as predicted by Eq. (12)) were clearly identified for specific short time intervals (Fig. 1b). A decrease in the time intervals (denoted $t_{\max }$ in this study) is noted, in which the theoretical curves show linear behavior with increasing bulk concentration for $a=1 \times 10^{-9} \mathrm{~mol} / \mathrm{cm}^{3}$. The data in Fig. $1 \mathrm{~b}$ indicate that $t_{\max }^{1 / 2}$ decreases from $80 \mathrm{~s}^{1 / 2}$ to $32 \mathrm{~s}^{1 / 2}$ as $C_{0} / a$ increases from 0.4 to 8 . In other words, $t_{\max }$ decreases from $6400 \mathrm{~s}$ to $1024 \mathrm{~s}$ as $C_{0} / a$ increases from 0.4 to 8 . A surface pressure (denoted $\pi_{\max }$ ) corresponding to $t_{\max }$ is therefore defined, in which the theoretical curves show a linear $\gamma$ vs. $t^{1 / 2}$ behavior. Fig. 2 shows the change in $t_{\max }$ as a function of surfactant concentration for surfactants with different surfactant activities, $a=1,5$, and $20\left(10^{-9} \mathrm{~mol} / \mathrm{cm}^{3}\right)$. For surfactants with different activities, $t_{\max }$ always decreases with bulk concentration. Moreover, a dramatic decrease occurs at a $C_{0} / a$ value of approximately 8 .

Fig. 3 details the deviation in $\gamma(t)$ between the tension data $\left(\gamma_{\text {data }}\right)$ predicted by the Ward-Tordai equation and those $\left(\gamma_{\text {approx }}\right)$ determined by the short-time approximation (Eq. (12)) at $t<t_{\max }$; $\Delta \gamma(t)=\gamma_{\text {data }}-\gamma_{\text {approx }}$. The data in Fig. 3 indicates for the case of $C_{0} / a=8$, a positive deviation occurs at the beginning, followed by a negative $\Delta \gamma(t)$; then, another positive value of $\Delta \gamma(t)$ occurs. The maximum deviation in $\gamma$ in this case is $\sim 0.7 \mathrm{mN} / \mathrm{m}$, which is roughly the measurement uncertainty in our experiments. Fig. 4 summarizes the relaxation of $\Delta \gamma(t)$ for different surfactant concentrations, $C_{0} / a=0.4-30$. At intermediate surfactant concentrations (2.2-8), the deviation in $\Delta \gamma(t)$ is similar to the behavior shown in Fig. 3. When the concentration is low $\left(C_{0} / a=0.4-2\right), \Delta \gamma(t)$ shows a small negative value, followed by a small positive one. In 

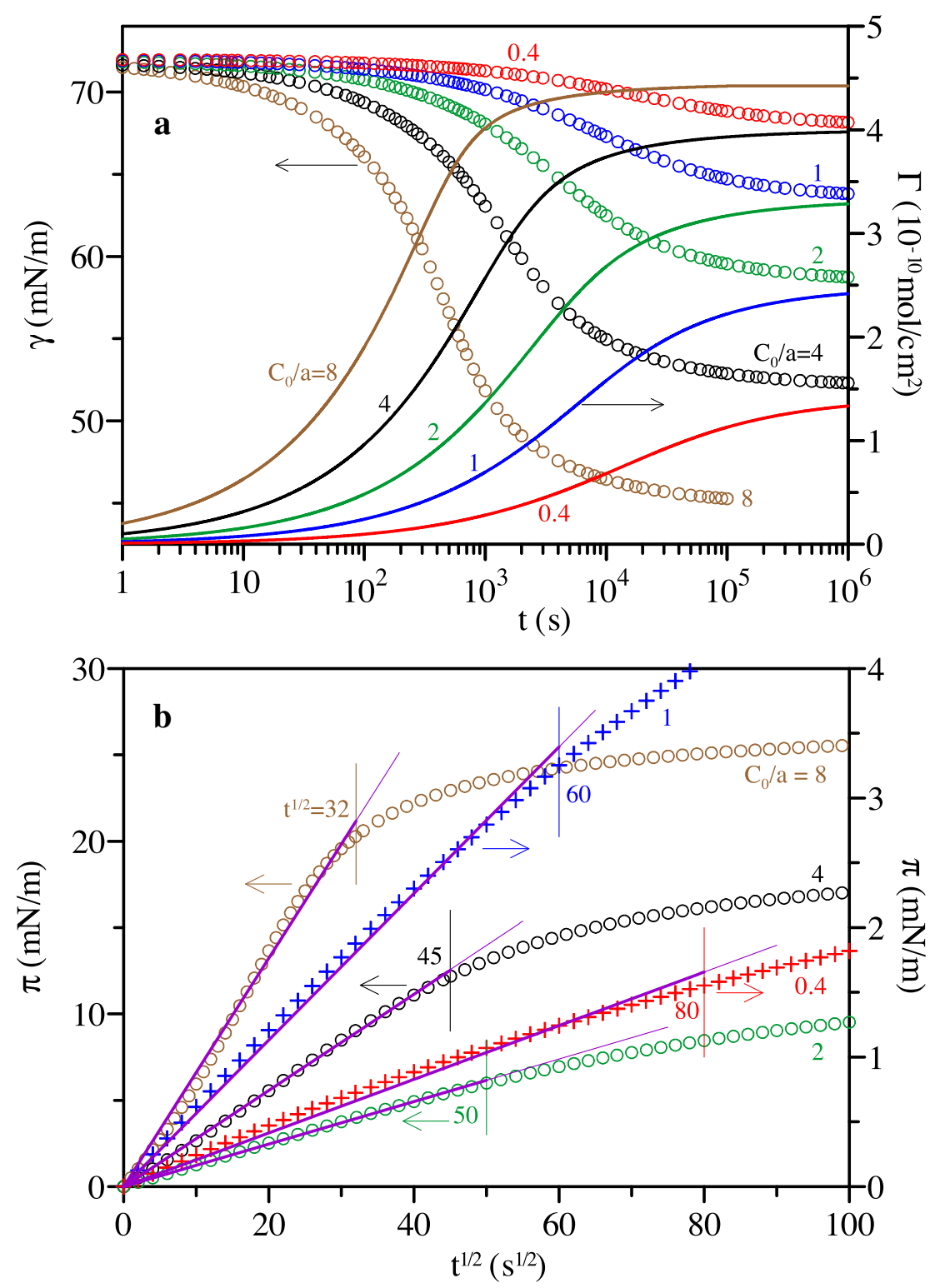

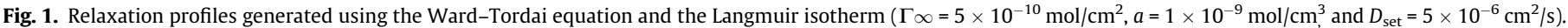
Profiles of (a) $\gamma(t), \Gamma(t)$ and (b) $\pi\left(t^{1 / 2}\right)$ for five different values of $C_{0} / a(=0.4,1,2,4,8)$.

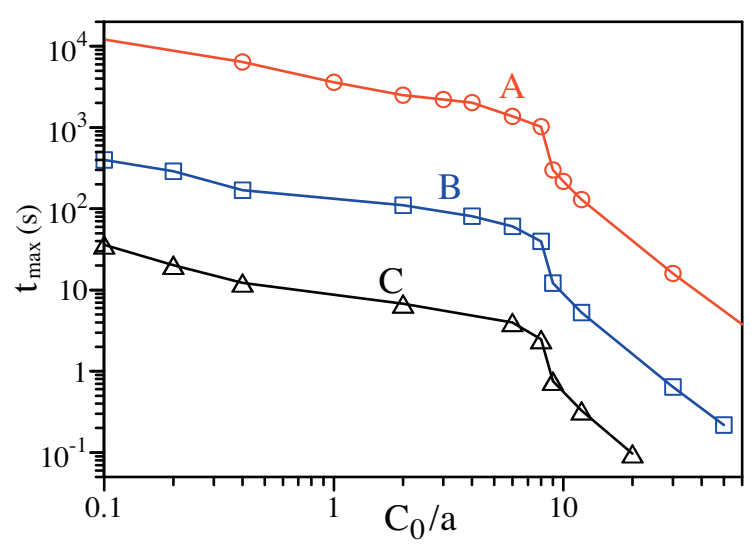

Fig. 2. Relaxation of $t_{\max }$ as a function of surfactant concentration $C_{0} / a$ and surfactant activity: $a=1(\mathrm{~A}), 5(\mathrm{~B})$, and 20 (C) $\left(10^{-9} \mathrm{~mol} / \mathrm{cm}^{3}\right)$.

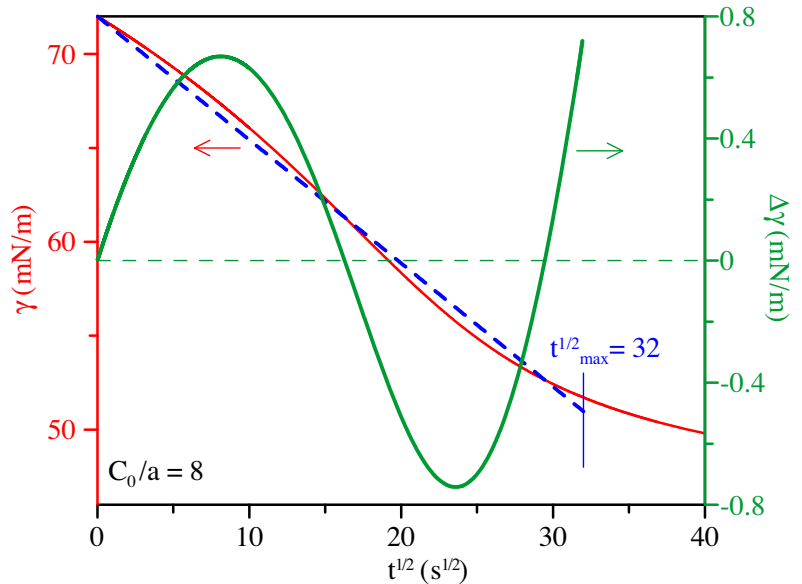

Fig. 3. Deviation in surface tension $\Delta \gamma(t)$ between the $\gamma(t)$ data (solid curve, $\gamma_{\text {data }}$ ) and the linear prediction (dashed line, $\gamma_{\text {approx }}$ ) of the short-time approximation for $C_{0} / a=8 ; \Delta \gamma(t)=\gamma_{\text {data }}-\gamma_{\text {approx }}$. 


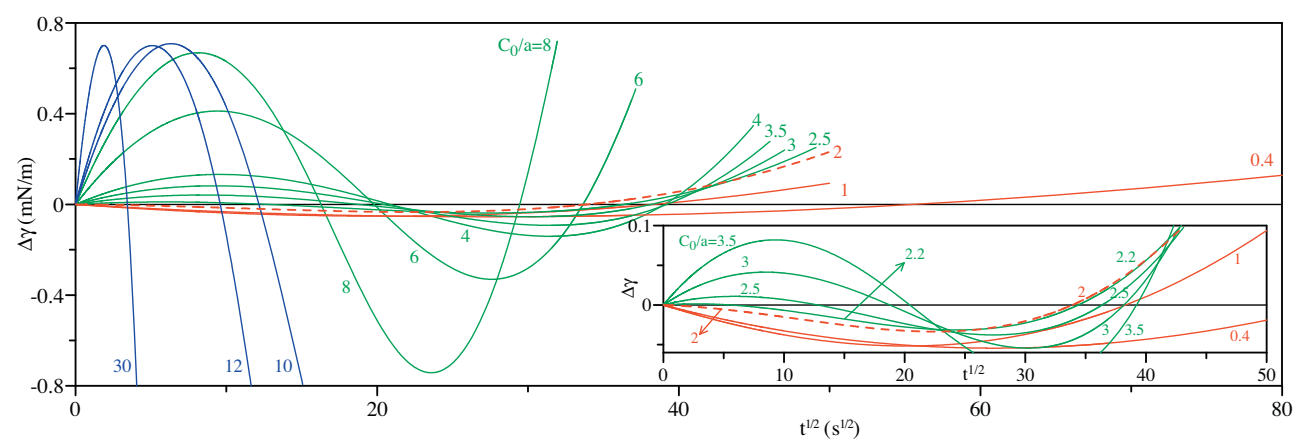

Fig. 4. Relaxation of the tension deviation $\Delta \gamma(t)$ at different surfactant concentrations $C_{0} / a$.

contrast, at high concentrations $\left(C_{0} / a>10\right)$, a significantly positive value of $\Delta \gamma(t)$ occurs first, followed by a negative one .

In applying the short-time approximation technique, experimental dynamic surface tension data are linearly fitted (lines 1 in Fig. 5) to estimate the surfactant diffusivity when an adsorption process is diffusion-controlled. Fig. 5 illustrates the fitting and the evaluation of the diffusivity $D$ using the short-time approximation technique for the early stages of the dynamic $\gamma(t)$ data derived from the Ward-Tordai equation. For surfactant concentrations $\left(C_{0} / a\right)$ lower than $\sim 2$ this linear fitting calculation leads to an underestimation of the diffusivity (Fig. $5 \mathrm{a}$ ), whereas for $C_{0} / a$ values greater than 2, $D$ is overestimated (Fig. 5 c). In both cases, the deviation from the established value $D_{\text {set }}$ increases with the time range considered such that, intuitively, shorter time intervals lead to more precise estimates of the diffusivity (lines 2 in Figs. 5a and c). Interestingly, as previously noted, for $C_{0} / a=\sim 2$, the deviations in the estimated $D$ (shown as the line 1 in Fig. $5 \mathrm{~b}$ ) from $D_{\text {set }}$ (shown as the line "2" in Fig. $5 \mathrm{~b}$ ) are negligible for all time ranges, where the $\gamma(t)-t^{1 / 2}$ curve follows a linear trend.

This deviation in the estimated value of $D$ (underestimation at low $C_{0} / a$, nearly zero deviation at $C_{0} / a=\sim 2$, and overestimation at large $\left.C_{0} / a\right)$ is derived from the deviation in the tension $\Delta \gamma(t)$ shown in Fig. 4. At low concentration, $\Delta \gamma(t)<0$ first, then $\Delta \gamma(t)>0$ over time (Figs. 4 and $5 \mathrm{a}$ ). The positive $\Delta \gamma(t)$ deviation causes a smaller $D$ value to be estimated by linear fitting (Eq. (12)); therefore, $D$ is underestimated. At high concentration, $\Delta \gamma(t)>0$ first, then $\Delta \gamma(t)<0$ over time (Figs. 4 and $5 \mathrm{c}$ ). The negative $\Delta \gamma(t)$ deviation causes a larger $D$ value to be estimated by linear fitting; therefore a $D$ is overestimated. At $C_{0} / a=\sim 2, \Delta \gamma(t)$ is small; therefore, the effects induced by positive and negative values of $\Delta \gamma(t)$ are balanced, resulting in a small deviation in $D$.

The data in Figs. 5a and c lead to another conclusion: a larger deviation in $D$ is observed when a larger range of $\gamma(t)$ data is used for the short-time approximation. Fig. $5 \mathrm{c}$ shows that $D=7.7 \times 10^{-6}$ $\mathrm{cm}^{2} / \mathrm{s}$ was obtained when the $\gamma(t)$ data at $t^{1 / 2}<20 \mathrm{~s}^{1 / 2}$ were used. When the $\gamma(t)$ data at $t^{1 / 2}<32 \mathrm{~s}^{1 / 2}$ were used, a larger deviation in $D\left(8.6 \times 10^{-6} \mathrm{~cm}^{2} / \mathrm{s}\right)$ was observed.

The effect of surfactant concentration $\left(C_{0} / a\right)$ on the deviation in $D\left(D_{\text {err }}=100\left(D_{\text {approx }}-D_{\text {set }}\right) / D_{\text {set }}\right)$ is shown in Fig. 6. A nearly linear dependence was observed when $D_{\text {err }}$ was plotted as a function of surface pressure $\pi$. The value of $\pi$ indicates the range of $\gamma(t)$ data used for linear fitting by the short-time approximation technique. This linear dependence confirms the conclusion discussed above: a larger deviation in $D$ is observed when a larger range of $\gamma(t)$ data is used. Fig. 6a also indicates that at high concentrations, a larger range of $\gamma(t)$ data can be used for evaluating $D$ in applying the short-time approximation technique. The last data point (solid circle) indicates the maximum range of $\gamma(t)$ data over which the tension deviation $\left[\Delta \gamma(t)=\gamma_{\text {data }}-\gamma_{\text {approx }}\right]$ is still allowable (less than the measurement uncertainty; $0.1 \mathrm{mN} / \mathrm{m}$ at low surfactant
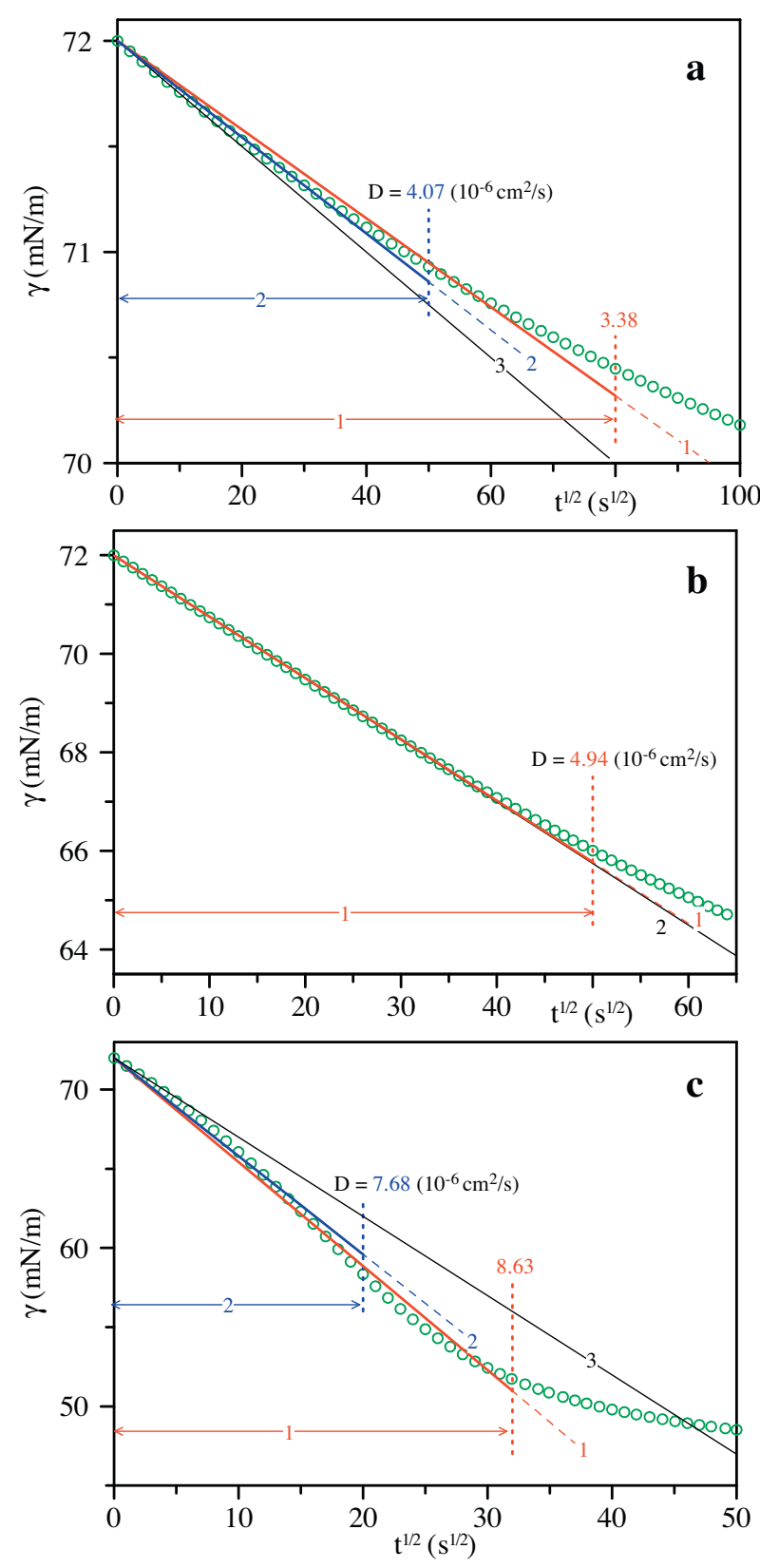

Fig. 5. The linear behavior, $\gamma(t)-t^{1 / 2}$, determined by the short-time approximation at different surfactant concentrations for the data shown in Fig. 1 (line 1): $C_{0} / a=0.4$ (a), 2.0 (b), and 8.0 (c). Lines 1 and 2 shows the effect of the time interval used on estimating the diffusivity (lines 2 use a short interval than lines 1 ). Line 3 shows $\gamma(t)$ derived from the short-time approximation with $D_{\text {set }}=5 \times 10^{-6} \mathrm{~cm}^{2} / \mathrm{s}$. 

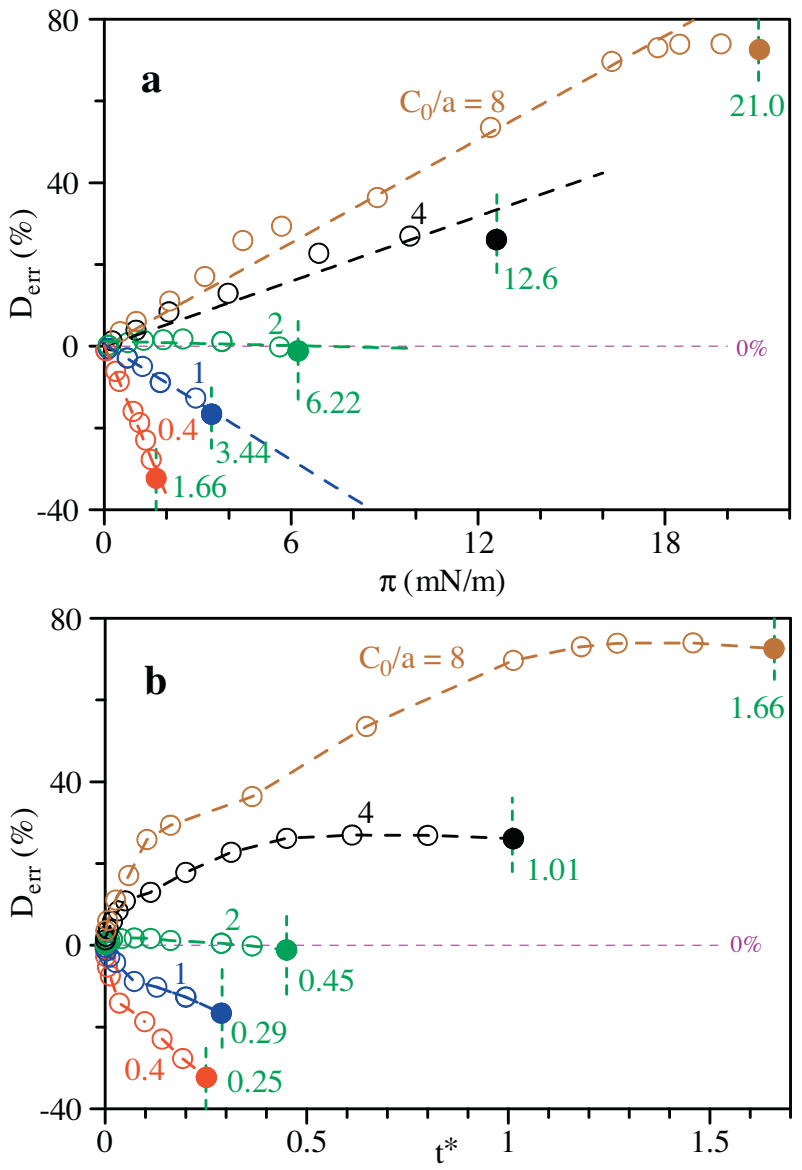

Fig. 6. Nondimensional diffusivity error $\left(D_{\text {err }}\right)$ as a function of (a) the surface pressure $(\pi)$ and (b) dimensionless time $\left(t^{*}\right)$ for $C_{0} / a=0.4,1,2,4$, and 8 .

concentrations and $0.7 \mathrm{mN} / \mathrm{m}$ at high concentrations in this study). The relationship between $D_{\text {err }}$ and dimensionless time, $t^{*}=t /\left[\Gamma_{\mathrm{eq}}^{2} /\left(C_{0}^{2} D\right)\right]$, at different surfactant concentrations is shown in Fig. $6 \mathrm{~b}$, in which $D_{\text {err }}$ levels off as $t^{*}$ increases at high concentrations.

To determine these general benchmarks, the surfactant diffusivity, estimated by the short-time approximation technique for different surfactant concentrations, was initially investigated as a function of the surface pressure, $\pi=\gamma_{0}-\gamma$. Fig. 7a shows the limiting conditions $\left(\pi_{\max }\right.$ ) for which the deviation between $\gamma_{\text {approx }}$ (from the short time approximation straight line) and $\gamma_{\text {data }}(t)$ (from the Ward-Tordai equation) is allowable $(\Delta \gamma(t)<0.1$ and $0.7 \mathrm{mN} / \mathrm{m}$ at low and high concentrations). Fig. 7a shows the region (below the curve of $\pi_{\max }$ vs. $\left.C_{0} / a\right)$ where the short-time approximation is applicable (i.e., with a reasonable $\Delta \gamma(t)$ ). The error in estimating the surfactant diffusivity may range from $-30 \%$ to $70 \%$ (Fig. 6), depending on the surfactant concentration $C_{0} / a$ and the region of $\gamma_{\text {data }}(t)$ used (indicating by $\pi$ or $t^{*}$ ). In order for the readers to apply the data (the region where the short-time approximation is applicable) easily, Fig. 7a are also shows this dependence in dimensionless parameters $\pi_{\max }^{*}\left(=\pi_{\max } \gamma_{0}-\gamma_{\mathrm{eq}}\right)$ and $t_{\max }^{*}\left(=t_{\max } /\left[\Gamma_{\text {eq }}^{2} /\left(C_{0}^{2} D\right)\right]\right)$ as a function of surfactant concentration $C_{0} / a$. Dimensionless time $t_{\max }^{*}$ indicates the time $t^{*}$ where $\pi^{*}$ reaches $\pi_{\max }^{*}$.

Interestingly, as illustrated in Fig. $7 \mathrm{~b}$, a linear dependence $\left(\pi_{\max }\right.$ vs. $\left.C_{0} / a\right)$ was observed at low surfactant concentrations $\left(C_{0} / a \leqslant 6\right)$. The existence of a linear dependence is probably because the surfactant concentration is low. It is noted that this linear dependence in term of dimensionless parameter ( $\pi_{\max }^{*}$ vs. $C_{0} / a$ and $t_{\max }^{*}$ vs. $C_{0} / a$ ) works only at $0.4 \leqslant C_{0} / a \leqslant 6$. At an extremely low concentration $\left(C_{0} / a \leqslant 0.4\right)$, both $\pi_{\max }^{*}$ and $t_{\max }^{*}$ decrease as concentration increases.
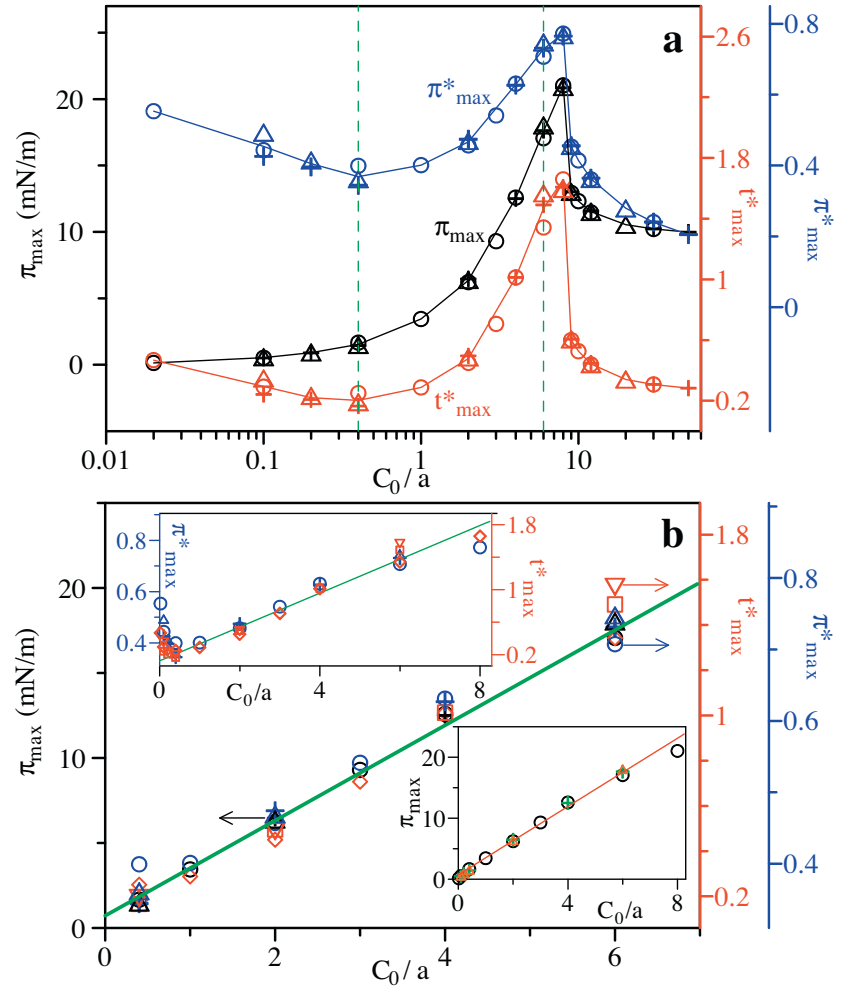

Fig. 7. (a) Conditions (maximal surface pressure $\pi_{\max }$, dimensionless time $t_{\max }^{*}$, and dimensionless surface pressure $\pi_{\max }^{*}$ ) under which the short-time linear approximation technique is applicable as a function of surfactant concentration $C_{0} / a$ for different surfactants with surfactant activities of $a=1(\bigcirc, \diamond), 5(+, \square)$ and $20(\Delta, \nabla)\left(10^{-9} \mathrm{~mol} /\right.$ $\mathrm{cm}^{2}$ ); (b) conditions for surfactant concentration $C_{0} / a \leqslant 6$.

A simple linear dependence can be used to determine the limiting working conditions of the short-time approximation:

$\pi_{\max }=2.80\left(C_{0} / a\right)+0.72 \quad[=] \mathrm{mN} / \mathrm{m}$

$t_{\max }^{*}=0.21\left(C_{0} / a\right)+0.12[=]-$

$\pi_{\max }^{*}=0.066\left(C_{0} / a\right)+0.33[=]-$

For surface pressures greater than this maximal surface pressure, $\pi>\pi_{\max }$, the dynamic surface tension curve deviates significantly from the linear region of the short-time approximation, and thus, the linear fitting of the tension could lead to significant errors in the calculated diffusivity. Therefore, Eq. (13) defines the range of applicability of the short-time linear approximation technique in terms of the surface pressure as a function of surfactant concentration $C_{0} / a$.

A further analysis was conducted to evaluate the possible effect of the surfactant activity on the nondimensional error in the diffusivity evaluated from the short-time approximation technique. As illustrated in Fig. 8, the $D_{\text {err }}-\gamma$ curves for different $a$ values $\left(a=1 \times 10^{-9}, 5 \times 10^{-9}\right.$ and $\left.20 \times 10^{-9} \mathrm{~mol} / \mathrm{cm}^{3}\right)$, but the same surfactant concentration $C_{0} / a$, present a distinct trend. It can therefore be concluded that the surfactant activity plays only a minor role in obtaining a valid diffusivity estimate.

\section{Discussion}

Based on the definition of the Ward-Tordai equation in Eq. (6) and the assumption that Eq. (9) is valid when surfactant backward diffusion can be assumed to be negligible, it is trivial to show how the deviation of $\gamma$ from the linear fitting is related to the actual 

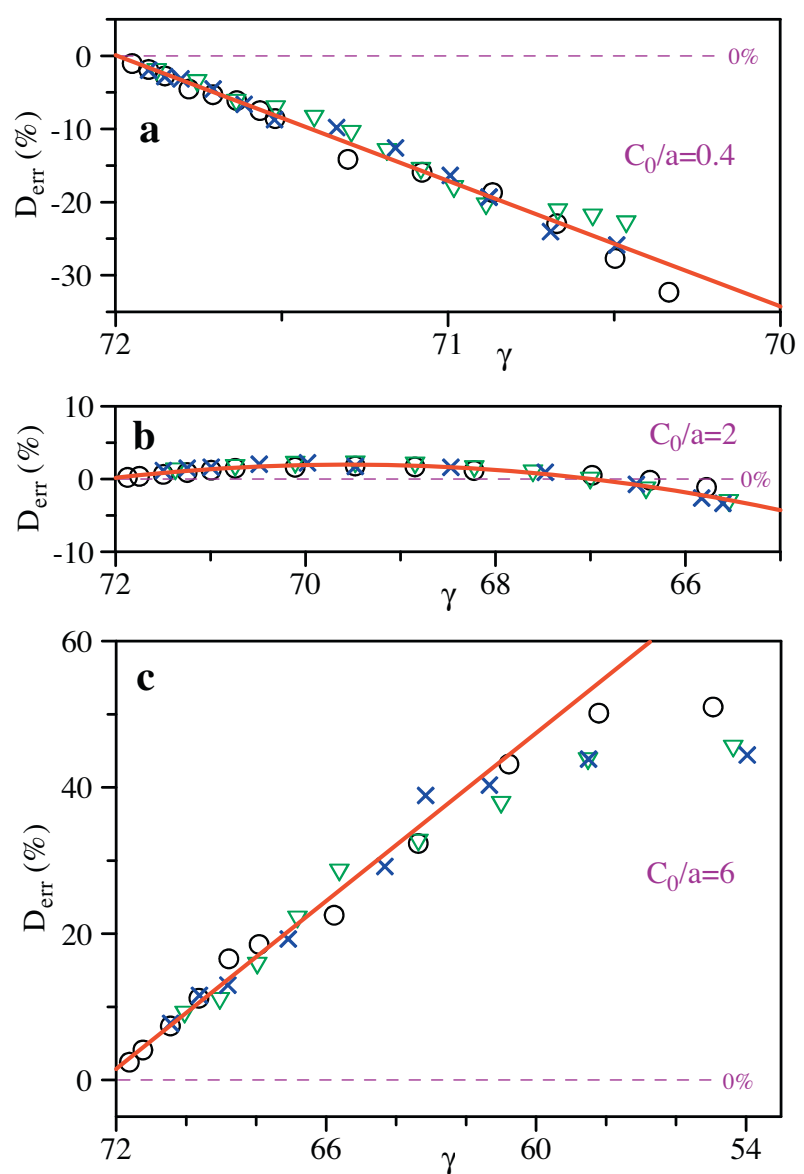

Fig. 8. A nearly unique nondimensional error in diffusivity $D_{\text {err }}$ evaluated from the short-time approximation technique as function of surface tension $[\gamma(t)$ range used for the linear short-time approximation fitting] for different surfactant activities $a=1(\bigcirc), 5(\nabla)$, and $20(\times)\left(10^{-9} \mathrm{~mol} / \mathrm{cm}^{3}\right)$ at surfactant concentrations of $C_{0} / a=0.4$ (a), 2 (b), and 6 (c).

balance between the forward and backward surfactant mass transport processes. To enhance understanding of this crucial aspect, both $\operatorname{Term}_{F}\left(=2 C_{0}(D t / \pi)^{1 / 2}\right.$, Fig. 9a, solid curves $)$ and $\operatorname{Term}_{B}$ $\left(=2(D / \pi)^{1 / 2} \quad \int_{0}^{\sqrt{t}} C_{S}(t-\tau) \mathrm{d} \sqrt{\tau}\right.$, Fig. 9a, dashed curves) of the Ward-Tordai equation were calculated, using the Langmuir isotherm $\left(\Gamma_{\infty}=5 \times\right.$

$10^{-10} \mathrm{~mol} / \mathrm{cm}^{2}, a=1 \times 10^{-9} \mathrm{~mol} / \mathrm{cm}^{3}$ and $D_{\text {set }}=5 \times 10^{-6} \mathrm{~cm}^{2} / \mathrm{s}$ ), for three different surfactant concentrations. As clearly shown in Fig. 9a, after a rather short initial time interval, the backward diffusivity can no longer be omitted. Indeed, it is worth noting that even for time ranges close to $t=0, \operatorname{Term}_{B}$ is not null and a small degree of backward diffusion occurs.

The relative importance of the backward diffusivity compared to the forward diffusivity is also clearly illustrated in Fig. 9b, in which the Term $_{B} /$ Term $_{F}$ ratio is analyzed: for time intervals $t>100$ s the diffusion of the surfactant from the sublayer to the bulk phase must be considered, and the use of the short-time linear approximation technique should not be considered to be completely accurate.

Finally, we compared all of the diffusivity data, estimated using the short-time linear approximation technique and reported in the literature, with the limiting criteria identified in this study. Fig. 10 shows the surface pressure $\pi$ as function of surfactant concentration $C_{0} / a$ for the data presented in Table 1 . The maximum error limiting criteria (solid curve) and the criteria indicating a 30\% error in diffusivity (dashed curve) were analyzed. It is noteworthy that the majority of the reported diffusivity data appear to be included
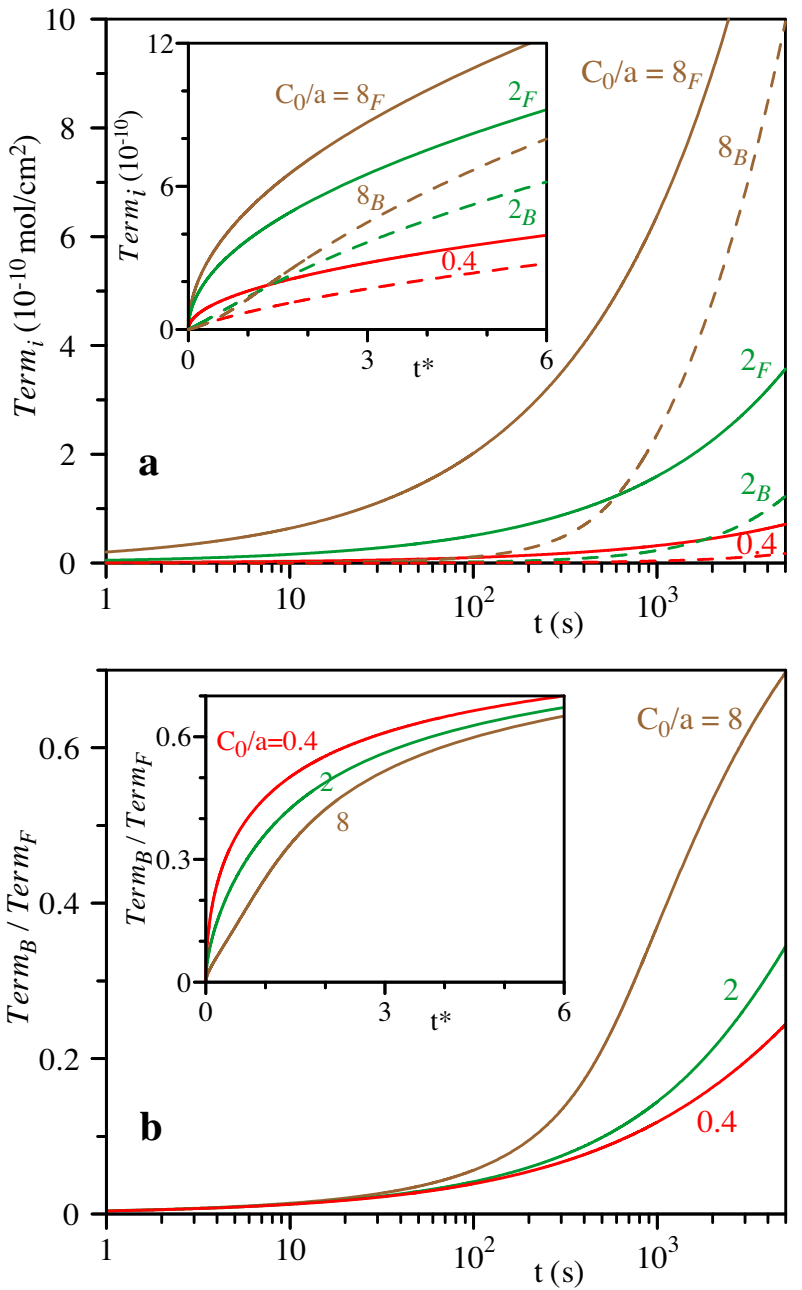

Fig. 9. (a) Forward diffusion (solid curves) and backward diffusion (dashed curves) determined from the Ward-Tordai equation and (b) the ratio of backward diffusion $\left(\right.$ Term $\left._{B}\right)$ to forward diffusion $\left(\right.$ Term $\left._{F}\right)$ as a function of time (main figure) and dimensionless time (inset); $t / t^{*}=25,500,5560$, and 617 for $C_{0} / a=0.4,2.0$ and 8.0, respectively.

in our error limiting criteria, with the following error range for the estimated diffusivity coefficient: $-30 \%<D_{\mathrm{err}}<30 \%$. Regarding the various data reported in the literature that were observed to fall above our maximum limiting criteria, special attention is required. Specifically, we do not claim that the surfactant diffusivities estimated by the authors who furnished these data are actually affected by errors to a great extent, but the fact that these points are far above our limit certainly suggests that the fundamental assumptions that led to our criteria (planar gas-liquid surfaces, diffusion-controlled mass transport process and Langmuir adsorption isotherm) may not be applied for these specific systems.

In this work, a fixed diffusivity $\left(D_{\text {set }}=5 \times 10^{-6} \mathrm{~cm}^{2} / \mathrm{s}\right)$ was set for all the theoretical simulation. This diffusivity was chosen because the most of small surfactants have a diffusivity closed to this value. It is noted that the choice of the $D_{\text {set }}$ won't make any change on the conclusion and phenomenon presented in this manuscript because the data was presented in the variation percentage $\left(D_{\text {err }}\right)$, as shown in Figs. 6 and 8.

This study considered only a planar air-water interface for surfactants following the Langmuir adsorption isotherm (i.e., no intermolecular interaction between the adsorbed surfactant molecules at surface). However, the evaluation error using the short time approximation method depends not only on surfactant concentration $\left(C_{0} / a\right)$, but also on the spherical curvature of air-water 


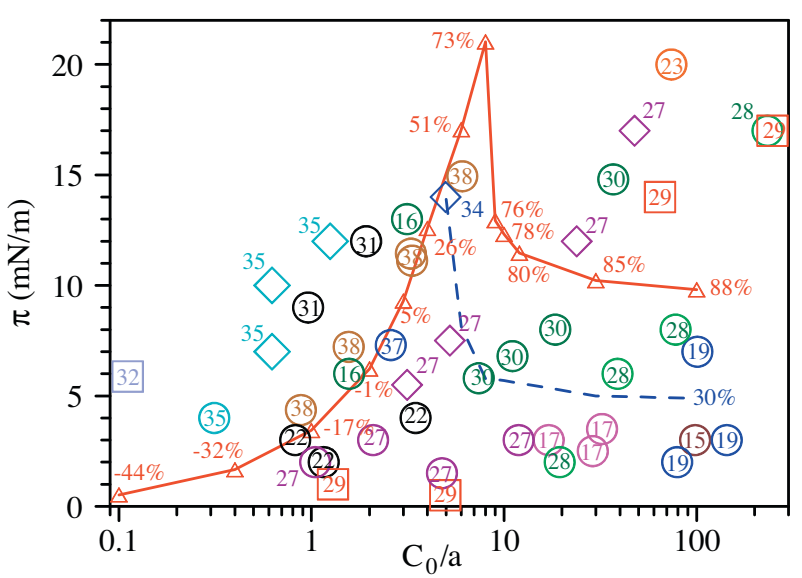

Fig. 10. Comparison between literature data (in Table 1) and limiting surface pressure $\pi$ criteria proposed in this work as function of $C_{0} / a$. The criteria are evaluated for maximum limiting criteria (solid curve) and for $-30 \%<D_{\mathrm{err}}<30 \%$ (dashed curve). Literature data shown in circles were obtained by pendant drop or maximum bubble pressure measurement methods, data shown in squares were obtained by the Wilhelmy plate method, and data shown in diamonds were obtained by the short-time linear approximation technique but without passing through the initial point of $\gamma_{0}=72 \mathrm{mN} / \mathrm{m}$ at $t=0$. The numbers shown near (or inside) the symbols indicate the reference articles. The numbers shown near the line indicate the theoretical percentage of diffusivity error.

interface and the intermolecular interaction force. The applicability criteria for these more complicated systems have been studied and in the course of the article writing in our laboratory for the time being.

\section{Conclusions}

The short-time approximation technique is widely used for linearly fitting experimental dynamic surface tension data to estimate the diffusivity and to determine the adsorption mechanism of a given process. However, because it is assumed that at the beginning of the adsorption process, surfactant backward diffusion from the sublayer to the bulk phase is negligible and $\sqrt{t} C_{S}$ is constant, the applicability of this technique is limited to specific time intervals.

Therefore, in this work, a theoretical numerical simulation of the short-time approximation technique was conducted, and general criteria for its accurate utilization were analyzed. Specifically, for systems assuming planar gas-liquid surfaces, a diffusion-controlled mass transport process and a Langmuir adsorption isotherm, some simple rules were established in terms of maximal surface pressure $\pi_{\max }$ and maximal dimensionless time $t_{\max }^{*}$ as a function of the dimensionless surfactant concentration $C_{0} / a$. For $\pi>\pi_{\max }$ or $t>t_{\max }^{*}$, the dynamic surface tension curve deviates from the linear region of the short-time approximation, and thus, the linear fitting of the surface tension could lead to significant errors in diffusivity estimates. Under these conditions, thus, the actual applicability of the short-time linear approximation method must be critically evaluated.

\section{Conflict of interest}

None declared.

\section{Acknowledgments}

The authors would like to express great appreciation to Taiwan NSC and the Italian National Research Council for the support within the project "Mixed Particle-Surfactant Layers at Liquid Interfaces" (NSC/CNR Bilater Agreement 2014-2015 and NSC-100-2221-E-011-101).

\section{References}

[1] B.E. Chistyakov, Theory and practical application aspects of surfactants, Surfactants Chem. Interfacial Prop. Appl. (2001) 511-618.

[2] Acmite Market Intelligence, Market Report: Global Surfactant Market, 2013.

[3] S.S. Dukhin, R. Miller, G. Kretzschmar, On the theory of adsorption kinetics of ionic surfactants at fluid interfaces, Colloid Polym. Sci. 269 (1991) 923-928.

[4] A.F.H. Ward, L. Tordai, Time-dependence of boundary tensions of solutions I. the role of diffusion in time-effects, J. Chem. Phys. 14 (1946) 453-461.

[5] R. Miller, A.V. Makievski, V.B. Fainerman, Dynamics of adsorption from solutions, Surfactants Chem. Interfacial Prop. Appl. (2001) 287-399.

[6] R. Defay, J.R. Hommelen III, The importance of diffusion in the adsorption process of some alcohols and acids in dilute aqueous solutions, J. Colloid Sci. 14 (1959) 411-418.

[7] R.S. Hansen, T.C. Wallace, The kinetics of adsorption of organic acids at the water-air interface, J. Phys. Chem. 63 (1959) 1085-1092.

[8] R. van den Bogaert, P. Joos, Dynamic surface tensions of sodium myristate solutions, J. Phys. Chem. 83 (1979) 2244-2248.

[9] C.D. Ampatzidis, E.-M.A. Varka, T.D. Karapantsios, Dynamic surface properties of eco-friendly phenylalanine glycerol ether surfactants at the W/A interface, Colloids Surf. A Physicochem. Eng. Asp. 441 (2014) 872-879.

[10] M. Eftekhardadkhah, P. Reynders, G. Øye, Dynamic adsorption of water soluble crude oil components at air bubbles, Chem. Eng. Sci. 101 (2013) 359-365.

[11] S. Ferdous, M.A. Ioannidis, D. Henneke, Adsorption kinetics of alkanethiolcapped gold nanoparticles at the hexane-water interface, J. Nanoparticle Res. 13 (2011) 6579-6589.

[12] T.D. Gurkov, Adsorption kinetics under the influence of barriers at the subsurface layer, Colloid Polym. Sci. 289 (2011) 1905-1915.

[13] K. Sakai, S. Umezawa, M. Tamura, Y. Takamatsu, K. Tsuchiya, K. Torigoe, et al., Adsorption and micellization behavior of novel gluconamide-type gemini surfactants, J. Colloid Interface Sci. 318 (2008) 440-448.

[14] A.M. Díez-Pascual, A. Compostizo, A. Crespo-Colín, R.G. Rubio, R. Miller, Adsorption of water-soluble polymers with surfactant character. Adsorption kinetics and equilibrium properties, J. Colloid Interface Sci. 307 (2007) 398404.

[15] T. Yoshimura, A. Sakato, K. Tsuchiya, T. Ohkubo, H. Sakai, M. Abe, Adsorption and aggregation properties of amino acid-based $\mathrm{N}$-alkyl cysteine monomeric and N, N'-dialkyl cystine gemini surfactants, J. Colloid Interface Sci. 308 (2007) 466-473.

[16] Y.A. Gao, W.G. Hou, Z.N. Wang, G.Z. Li, B.X. Han, G.Y. Zhang, Dynamic surface tensions of fluorous surfactant solutions, Chinese J. Chem. 23 (2005) 362-366.

[17] C. Delgado, M.D. Merchán, M.M. Velázquez, S. Pegiadou, L. Pérez, M. Rosa Infante, The adsorption kinetics of 1-N-l-tryptophan-glycerol-ether surfactants at the air-liquid interface. effect of surfactant concentration and alkyl chain length, Colloids Surfaces A Physicochem. Eng. Asp. 233 (2004) 137144.

[18] J.M. Rodriguez Patino, C. Carrera Sanchez, S.E. Molina Ortiz, R. Rodriguez Nino, C. Anon, Adsorption of soy globulin films at the air-water interface, Ind. Eng. Chem. Res, 43 (2004) 1681-1689.

[19] U.R.M. Kjellin, J. Reimer, P. Hansson, An investigation of dynamic surface tension, critical micelle concentration, and aggregation number of three nonionic surfactants using NMR, time-resolved fluorescence quenching, and maximum bubble pressure tensiometry, J. Colloid Interface Sci. 262 (2003) 506-515.

[20] E. Alami, K. Holmberg, J. Eastoe, Adsorption properties of novel gemini surfactants with nonidentical head groups, J. Colloid Interface Sci. 247 (2002) 447-455.

[21] R. Rodriguez Nino, J.M. Rodriguez Patino, Effect of the aqueous phase composition on the adsorption of bovine serum albumin to the air-water interface, Ind. Eng. Chem. Res. 41 (2002) 1489-1495.

[22] M.G. Munoz, F. Monroy, F. Ortega, R.G. Rubio, D. Langevin, Monolayers of symmetric triblock copolymers at the air-water interface. 2. adsorption kinetics, Langmuir 16 (2000) 1094-1101.

[23] B.V. Zhmud, F. Tiberg, J. Kizling, Dynamic surface tension in concentrated solutions of CnEm surfactants: a comparison between the theory and experiment, Langmuir 16 (2000) 2557-2565.

[24] N. Wu, J. Dai, F. Micale, Dynamic surface tension measurement with a dynamic Wilhelmy plate technique, J. Colloid Interface Sci. 215 (1999) 258-269.

[25] J. Eastoe, J.S. Dalton, R.K. Heenan, Dynamic surface tensions and micelle structures of dichained phosphatidylcholine surfactant solutions, Langmuir 14 (1998) 5719-5724

[26] M. Ferrari, L. Liggieri, F. Ravera, Adsorption properties of C10E8 at the waterhexane interface, J. Phys. Chem. B. 102 (1998) 10521-10527.

[27] E.V. Aksenenko, A.V. Makievski, R. Miller, V.B. Fainerman, Dynamic surface tension of aqueous alkyl dimethyl phosphine oxide solutions. Effect of the alkyl chain length, Colloids Surfaces A Physicochem. Eng. Asp. 143 (1998) 311 321.

[28] J. Eastoe, J.S. Dalton, P.G.A. Rogueda, E.R. Crooks, A.R. Pitt, E.A. Simister, Dynamic surface tensions of nonionic surfactant solutions, J. Colloid Interface Sci. 188 (1997) 423-430.

[29] R. Wüstneck, J. Krägel, R. Miller, V.B. Fainerman, P.J. Wilde, D.K. Sarker, et al., Dynamic surface tension and adsorption properties of $\beta$-casein and $\beta$ lactoglobulin, Food Hydrocoll. 10 (1996) 395-405.

[30] J. Li, R. Miller, H. Mohwald, Characterisation of phospholipid layers at liquid interfaces.1. Dynamics of adsorption of phospholipids at the chloroform/water interface, Colloids Surfaces A Physicochem. Eng. Asp. 114 (1996) 113-121. 
[31] V.B. Fainerman, R. Miller, Dynamic surface tension measurements in submillisecond range, J. Colloid Interface Sci. 175 (1995) 118-121.

[32] G. Geeraerts, P. Joos, F. Villé, Dynamic surface tensions and dynamic surface potentials of aqueous solutions of a cationic surface active electrolyte, Colloids Surfaces A Physicochem. Eng. Asp. 95 (1995) 281-291.

[33] V.B. Fainerman, A.V. Makievski, P. Joos, Adsorption kinetics of octylphenyl ethers of poly(ethylene glycol)s on the solution-air interface, Colloids Surfaces A Physicochem. Eng. Asp. 90 (1994) 213-224.

[34] V.B. Fainerman, A.V. Makievski, R. Miller, The analysis of dynamic surface tension of sodium alkyl sulphate solutions, based on asymptotic equations of adsorption kinetic theory, Colloids Surfaces A Physicochem. Eng. Asp. 87 (1994) 61-75.

[35] J.P. Fang, P. Joos, The dynamic surface tension of SDS-dodecanol mixtures 1. The submicellar systems, Colloids Surf. 65 (1992) 113-120.

[36] D.V. Dass, H.J. Van Enckevort, A.G. Langdon, Protein adsorption by colloidal particles, J. Colloid Interface Sci. 116 (1987) 523-531.

[37] R. van den Bogaert, P. Joos, Diffusion-controlled adsorption kinetics for a mixture of surface acthe agents at the solution-air interface, J. Phys. Chem. (1980) 190-194.
[38] P. Joos, E. Rillaerts, Theory on the determination of the dynamic surface tension with the drop volume and maximum bubble pressure methods, J. Colloid Interface Sci. 79 (1981) 96-100.

[39] M. Blank, B.B. Lee, J.S. Britten, Adsorption kinetics of ovalbumin monolayers, J. Colloid Interface Sci. 50 (1975) 215-222.

[40] G.L. Gaines, G.W. Bender, Surface concentration of a styrene-dimethylsiloxane block copolymer in mixtures with polystyrene, Macromolecules 5 (1972) 8286.

41] R.L. Bendure, Dynamic surface tension determination with the maximum bubble pressure method, J. Colloid Interface Sci. 35 (1971) 238-248.

[42] S.Y. Lin, K. Mckeigue, C. Maldarelli, Effect of cohesive energies between adsorbed molecules on surfactant exchange processes: shifting from diffusion control for adsorption to kinetic diffusive control for re-equilibration, Langmuir 10 (1994) 3442-3448.

43] R. Miller, G. Kretzschmar, Numerische Lösung für ein gemischtes Modell der diffusions-kinetik-kontrollierten Adsorption, Colloid Polym. Sci. 258 (1980) $85-87$. 